\title{
Co-creating an Open Government Data Driven Public Service: The Case of Chicago's Food Inspection Forecasting Model
}

\author{
Keegan McBride, Gerli Aavik, Tarmo Kalvet, Robert Krimmer \\ Tallinn University of Technology, Tallinn, Estonia \\ \{Keegan.McBride | Gerli.Aavik | Tarmo.Kalvet | Robert.Krimmer@ttu.ee\}
}

\begin{abstract}
Large amounts of Open Government Data (OGD) have become available and co-created public services have started to emerge, but there is only limited empirical material available on co-created OGDdriven public services. To address this shortcoming and explore the concept of co-created OGD-driven public services the authors conducted an exploratory case study. The case study explored Chicago's use of $O G D$ in the co-creation of a predictive analytics model that forecasts critical safety violations at food serving establishments. The results of this exploratory work allowed for new insights to be gained on cocreated OGD-driven public services and led to the identification of six factors that seem to play a key role in allowing for a OGD-driven public service to be cocreated. The results of the initial work also provide valuable new information that can be used to aid in the development and improvement of the authors' conceptual model for understanding co-created $O G D$ driven public service.
\end{abstract}

\section{Introduction}

In current e-Government literature, there are two topics that have been receiving increased interest and focus: open government data (OGD) and co-creation [1]. Increasing evidence is appearing on OGD's benefits and potential [2] as well as the barriers preventing its usage [3], [4]. The second topic, cocreation, emerges from the concept of Coproduction, brought into the spotlight by Elinor Ostrom in 1972 [5]. A previous paper has linked these two topics and discussed the idea of a "co-created OGD-driven public service" [6]. This concept emerges from a new understanding of what a public service is, "public services are any services which are offered to the general public with the purpose of developing public value, regardless of the role that the public sector plays in the process" [6], [7].

The co-created OGD-driven public service has two main components. Firstly, when talking about the co- creation of new public services, co-creation may be understood as the involvement of outside, non-typical, stakeholders in the initiation, design, implementation, and evaluation of the public service [6]. There is a difference between coproduction and co-creation, this was highlighted in a recent work where it was stated that "all public services are coproduced, but not all public service are co-created" [8]. In co-created OGDdriven public services, the process in which cocreation takes place must be understood, for this purpose the framework put forth by [6] is used to provide an initial understanding.

Another interesting concept that allows a bridge to be built between the concepts of OGD and co-creation is the notion of Government as a Platform (GaaP). $\mathrm{GaaP}$ as a means for understanding the relationship between OGD and co-creation was brought forth by Linders in [9]. The core idea behind GaaP is that there is a large amount of governmentally held and generated data, dissemination of said data is becoming less difficult, and that this data is able to aid and drive the creation of new and innovative activities [9]. In the GaaP model, the government is providing OGD and this data may be used or exploited by any actor or stakeholder to create public value. This use and exploitation of the data may be understood as cocreation as the government is providing the data and, if the resulting applications produce public value, a new public service has been driven by OGD and was co-created.

There has been a clear increase in interest in the topics of co-creation and OGD, and some authors have worked on further conceptualizing the relationship between the two ideas as well as provided some understanding of how co-created OGD-driven public services may come into being [10]. However, currently, there is limited empirical work that looks at, and examines, co-created OGD-driven public services in the real world. There are two reasons for this, the first is because it is a relatively new concept, and the second is due to a general lack of real-world examples of co-created OGD-driven public services. This is an interesting research gap and it was further explored by 
conducting an analysis of data-analytics and OGD programs; an empirical example has the potential to aid and assist the current understanding of co-created OGD-driven public services. Because of this analysis and exploration, an interesting example made itself known. The service involved multiple stakeholders (city governmental agencies, private sector companies, NGOs, and citizens), was developed using open source code and is still freely available, heavily utilized OGD, and convincingly produced public value. Additionally, previous work has been done on OGD in the selected city that demonstrated the effectiveness of the OGD portal there [11]. This combination of factors seemed to allow the service to be titled a co-created OGD driven public service and it was thus selected for further analysis.

This paper aims to explore Chicago's use of OGD for a new predictive analytics model that allows the Chicago's Department of Public Health (CDoPH) to forecast critical safety violations at food serving establishments. Because of the exploration, new insight has been gained that can later be used to further develop the understanding of co-created OGD-driven public services. The importance and relevance of this case was summed up in a succinct manner by Tom Schenk, the Chief Data Officer of Chicago, in a report he authored on the service: "collaboration was a key component of this project... and each variables used in the model was available on Chicago's open data portal" [12]. Later in the report it was stated that "the portal was an effective tool to allow collaborative research", and that "this project was able to leverage Chicago's key data assets: its large volume of data, the transparency and size of its open data portal, and its ability and willingness to conduct research to improve city services, introduce savings, and increase engagement with Chicago-area businesses" [12]. The stakeholders involved in this collaborative effort were Chicago's Department of Innovation and Technology (CDoIT), members of Allstate Insurance's Data Science Team, CDoPH, the Civic Consulting Alliance (CCA), and, finally, citizens also have played a role in structuring the new public service.

In order to understand better the process of cocreated OGD-driven public services, an exploratory case study was conducted. This paper presents the case, reflects on the process, and discusses how the findings from the case grow and aid the current understanding of co-created OGD-driven public services. The paper is structured as follows. Chapter 2 will provide a brief overview of the methodology that was used to conduct the case study; this will be followed by a presentation of the case in Chapter 3 . Chapter 4 will discuss the findings that have emerged from the case. During the discussion, initial propositions will also be put forth to reflect back on the current theory and our understanding of co-created OGD-driven public services. Finally, in Chapter 5, the paper will be concluded and avenues for future research will be put forth.

\section{Methodology and Conceptual Model}

In the previous section, the case was briefly introduced. It was stated that the model utilizes multiple sources of OGD, and that collaboration between many different stakeholders was key for this model to be completed and implemented. It has also been said that the OGD portal is what allowed these different stakeholders to come together and exploit OGD to co-create this new OGD-driven public service. For these reasons, the Chicago food predictive analytics model was selected as the case for this paper. This holistic exploratory case study [13] aims to explore the process that was undergone to move the co-created OGD-driven public service from ideation through development and into its current stage. Though this may be defined as a critical and unique case, it is still only one case thus providing a lower level of generalizability. However, it should still allow an initial study to be conducted that provides insight into the inner workings of a co-created OGD-driven public service.

For the initial understanding of OGD-driven public service co-creation, the framework presented by [6], will be used. The aim of this paper is to explore a co-created OGD-driven public service and gain new insight, but the model is presented as it allows for a starting point in looking at co-created OGD-driven public services. Observing the process of the case at hand allow new insights to be gained in regards to what factors influence the co-creation of OGD-driven public services, potentially provide new insights into the conceptual understanding of co-created OGDdriven public services, and look at the different roles stakeholders played in this process. This new insight may then be used in future development and improvement of the model. In order to gain initial insight into the case, newspaper articles, source code, and a report on the model's GitHub page were consulted. With an initial foundational understanding in place, semi-structured interviews were conducted to delve into the case and understand better the dynamics at play.

Six semi-structured interviews were conducted with stakeholders representing different parties; one 
person was interviewed from CDoIT, CCA, Allstate, Montgomery County, whereas two members were interviewed from the CDoPH. These interviews were conducted during April and May 2017 over the phone or through Skype and lasted from between 15 to 40 minutes each; all interviews were recorded and then transcribed. The first interview conducted was with Tom Schenk, the main person behind the case, and then, using snowballing, other interviewees were selected. The interviewee from Montgomery County Department of Innovation was selected due to the county's relationship with the project (Montgomery County implemented Chicago's code with the help of a private sector partner), though they were not directly involved in the initial model development.

The interview questions aimed to provide a better understanding of the interviewee's role in the project, how they got involved, how the process unfolded, what went well and what did not go well, and then at the end participants were asked to add in any comments that were not discussed during the interview. The responses from the interviewees are presented and discussed in section 4, commonly mentioned themes and facts will be further used to draft initial propositions on what seems to influence the success of a co-created OGD-driven public service, as well as what factors seem to be needed to allow OGD-driven public service co-creation to take place.

\subsection{Conceptual Model}

A recent paper [6], has proposed that public services can be created through an innovation process based on the ideas of co-production and agile development. The model, shown in Figure 1, argues that in an environment where OGD and tools for data analytics, exploitation, and co-production are made widely available, any actor can take the lead and initiate or co-create data-driven services that create public value. To do that, it is important to focus on service user, be agile, develop quickly, listen to the service user, and be able to adapt quickly to changing needs. This service innovation process can be summarized through four points:

1. The government and citizens should be partners at all stages from ideation to creation to implementation of the new data-driven public service.

2. There should be an initial release of the public service at an early stage, or an 'MVP' of the public service, which allows the cycle to be started as quickly as possible.

3. The public service should be able to respond to user feedback from the initial launch.

4. User input should be sought and utilized at all stages of the public service creation.

The model argues that the traditional governmentdriven top-down waterfall-like method of public service production no longer meets the needs and expectations of the citizens and new collaborative and data-driven approaches are the way to go. The model follows a four-phase cycle of open government data driven co-initiation, co-design, co-implementation and co-evaluation.

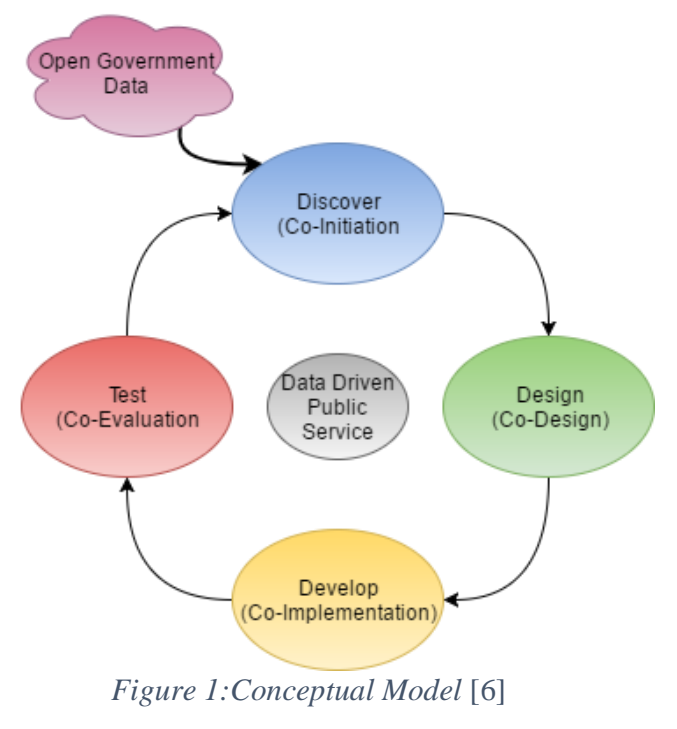




\section{The Case}

This section will present the case of the Chicago predictive food analytics model. It starts in section 3.1 by presenting the relevant contextual information surrounding the case. Following this, in section 3.2, the process of the development and implementation of the case will be discussed in two stages, the initial iteration (3.2) and the second iteration (3.3); these sections will also include the role of stakeholders, the processes of development, and the role of OGD and co-creation). The final aspect of the case to be presented in 3.4 is the impact of the new co-created OGD-driven public service and potential direction for the future.

\subsection{Context}

The context surrounding the case must be presented so that the case may be better understood. When looking at the relevant contextual factors for this case, there seems to be four main variables: access to a functioning OGD portal, previous experience in the realm of predictive analytics, a grant received from Bloomberg Philanthropies Mayors' Challenge, and a law requiring the inspection of establishments that serve food. These factors form the core contextual foundation for the case and their importance is presented in the following paragraphs

Chicago's OGD portal was initially developed in 2010, but in 2012 its importance was reinforced by an order issued by Mayor Rahm Emanuel. This order stated that Chicago must establish and maintain an OGD portal, and that every city agency must "make available online... at a level of granularity acceptable to DOIT (Department of Innovation and Technology), all appropriate datasets and associated metadata under such agency's control" [14]. When discussing the motivation for this executive order, the Mayor explained that OGD could be used to "create application that will improve service delivery and lead to greater quality of service for residents and more public engagement in City government". The OGD portal has since grown rapidly and currently provides access to over 550 datasets, applications built by the city and private developers, provides tutorials on how the available data may be exploited or analyzed, provides tools that allow for easy visualization of data, and has been visited over 38 million times [15]. This portal is run and maintained by the CDoIT.

In the introduction it was discussed how the concept of GaaP allows us to understand the relationship between OGD and co-creation. Interestingly, this was also pointed out by Brett
Goldstein, former Chief Data Officer of Chicago, where he stated that the idea of GaaP is a core part of the success of the Chicago OGD portal as they are able to "be the platform... and support the innovative ideas cultivated by various communities" [16].

Bloomberg Philanthropies organized a competition that would "inspire American cities to generate innovative ideas that solve major challenges and improve city life - and that ultimately can be shared with other cities to improve the wellbeing of the nation" [17]. The City of Chicago entered this competition and was awarded a grant for one million USD to develop a new "SmartData" platform that would allow government agencies easier access to predictive analytics tool; one condition of this grant was that all software developed would be open source [18]. Specifically, Chicago was selected to "create an open-source platform to harness the power of data to understand underlying trends and better direct limited resources" [17]. This grant provided the CDoIT funding to begin to undertake more ambitious OGDdriven predictive analytics models.

One of the initial models that emerged from the CDoIT was a model that could be used to predict when and where outbreaks of rodents would occur so that these outbreaks could be prematurely stopped [19]. This model was developed in cooperation with Carnegie Mellon University's Event and Pattern Detection Laboratory and then was put into production by Chicago's Department of Streets and Sanitation [19]. The model was well known throughout the City government agencies, and was cited by some of the interviewees as being one reason they were willing to participate in and allow Chicago's predictive food analytics model to be developed.

The final contextual factor to present is the legal requirements for inspecting establishments that serve food. The CDoPH's Food Protection Division is required to perform inspections of establishments that serve food, this authority comes from the City of Chicago's Food Service Sanitation Municipal Code and the Rules and Regulations promulgated by the Chicago Board of Health [20]. At the time of writing this article there were around 16,000 food establishments in the City of Chicago (there was over 15,000 when the predictive food analytics model was initially developed) [20]. These establishments have different requirements, but, generally, food establishments within the city must be inspected twice a year to make sure that they are incompliance with the aforementioned regulations on food safety. When inspections are carried out, one of the most important findings is whether a critical violation is taking place. Critical violations are those that have a high chance of starting or spreading food borne illnesses; the presence 
of a critical violation leads to a failure, the violation must then be fixed and the establishment re-inspected and reapproved by the $\mathrm{CDoPH}$ [12]. The results of these food inspections are also freely available on the Chicago OGD portal.

\subsection{Initial Development}

The City of Chicago and the CDoIT wanted to continue to expand their use of OGD and predictive analytics, thus increasing the efficiency of some agencies' day-to-day operations and provide increased public value. In order to do this, an initial list of potential use cases where OGD-driven predictive analytics capabilities could be used was drafted in 2014. Though the City of Chicago was interested in OGD and predictive analytics, the CDoIT still lacked a full data science team, thus outside help was needed. In order to find this outside help, the CDoIT reached out to a local organization, the CCA. The CCA is an organization that aims to improve the quality of life in Chicago by bringing together stakeholders from public, private, and non-profit sectors to work on new and innovative solutions for problems facing the city; the CCA roughly provides "fifteen million USD in pro bono services every year" [21]. The CCA had relations with the data science team at Allstate Insurance and approached them with the list of potential use cases from the City of Chicago. The members of the Allstate Data Science Team had experience with the Chicago OGD portal and knew that there was large amounts of data on food inspections within the city. The members also had a direct interest in the topic of food safety as they lived in the City of Chicago and thought it would be interesting to try to improve the food safety of the food serving establishments within the City. Thus, they got back to the CCA and said that they would be willing and interested to work on developing a predictive analytics model for the CDoPH's food inspections.

The policy that allowed for members of Allstate's Data Science Team to participate in this pro bono project is quite interesting; the company's "bluelight" policy allowed employees to spend up to $10 \%$ of their working time on pro-bono data science projects [22]. The logic behind this policy is that working on nontypical or new projects will boost their employees' skillset and expose them to new tools and technologies, ultimately benefiting Allstate, the Employees, and the Partner(s) receiving their assistance.

During 2014 when the initial development began, the City of Chicago had over 15,000 food establishments, to inspect these establishments the city had 36 food inspectors, and these establishments needed to be inspected twice a year, some establishments had to be inspected less and some more, but twice a year seems to be the general rule. This roughly translates to about one inspector for every 470 food establishments, due to the high workload and lack of optimization many critical food violations were going unnoticed or were being detected too late to stop or prevent outbreaks from starting/spreading [12]. Though there was a logic to how food inspectors were assigned, it was believed by the CDoIT that this process could be improved and made more effective through the adoption of an OGDdriven predictive analytics model. Though originally hesitant, the head of the CDoPH was willing to test a newly developed model as she had heard about the success of a previous model; this was the model mentioned previously in section 3.1.

To begin, the CCA organized meetings between the relevant parties and acted as a project manager (Allstate, CDoIT, and CDoPH). At these meetings, the business requirements of the $\mathrm{CDoPH}$ were discussed and presented to the developers and data scientists. Allstate's team ended up using multiple variables from Chicago's OGD portal and constructed a General Linear Model that would allow the highest risk food establishments to be inspected first. In essence, the model works by predicting what food establishments are the most likely to have a critical food violation, and then assigns these establishments to be inspected first; previously these assignments had been made following a business and risk based approach, but it still seemed somewhat random and inefficient. However, due to a misunderstanding of one variable, the first iteration of the model ended up being incorrect and needed to be adjusted.

This failure ended up being a major learning point for all involved stakeholders and emphasized the importance of communication early on as well as the importance of continuously communicating throughout development.

\subsection{Second Iteration}

Though the first implementation was not successful, it was improved upon and the misunderstanding was addressed by the CDoIT and CDoPH. This second attempt at the predictive analytics model is open source and the code is freely available on GitHub.

The model was tested over a two-month period (September and October 2014), during this time assignments were given out following normal operations, but the model was running simultaneously to see how it would compare to normal operations. 
After the testing had been completed and validated, the model was made operational by February of 2015.

When looking at the model, many different predictive features were tested, but currently the following nine predictors are utilized by the model:

1. "Establishments that had previous critical or serious violations.

2. Three-day average high temperature.

3. Nearby garbage and sanitation complaints.

4. The type of facility being inspected.

5. Nearby burglaries.

6. Whether the establishment has a tobacco license or has an incidental alcohol consumption license.

7. Length of time since last inspection.

8. The length of time the establishment has been operating.

9. And the assigned Inspector." [12].

The new code also utilized different predictive classification models, such as random forest, to try to get better results.

The predictive food analytics model uses the aforementioned predictors to classify which food establishments are the most likely to have a critical food violation. The individual in charge of assigning food inspectors to establishments accesses the predictions through a Shiny Application (Shiny is a package in $\mathrm{R}$ that allows for easy development of web pages and user interfaces). Food inspectors are then assigned to establishments that have been predicted or put forth by the model. The CDoIT GitHub page for the predictive food analytics model put forth Figure 2 to demonstrate better how the model works. In essence, food establishments with the highest risk of critical violations are inspected first.

Optimizing inspections for faster delivery

$\begin{array}{cc}\begin{array}{c}\text { Typical order of } \\ \text { inspections }\end{array} & \begin{array}{c}\text { Data-driven order } \\ \text { of inspections }\end{array}\end{array}$

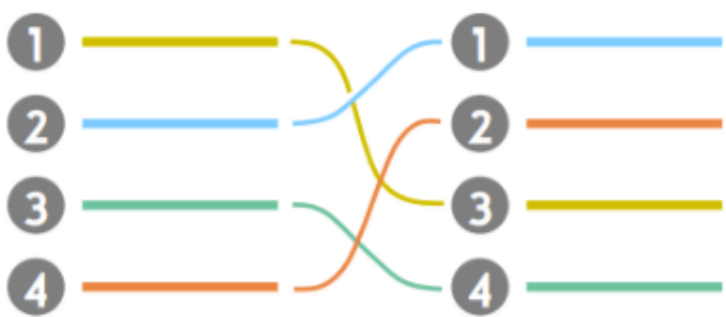

Figure 2: Optimized food inspection process [12]

\subsection{Impact and Future}

In 2014, the model was trained and evaluated over a two-month period. The results from the model were compared to the results of the actual food inspections occurring at the same time. This comparison allowed a clear advantage to be seen if the data-driven model had been used instead of the traditional approach. The model had allowed for critical food violations, on average, to be found 7.5 days earlier [12]. Thus, this would allow for potential food borne illness outbreaks to be prevented, or have their severity limited, as the violations responsible were being caught and addressed earlier. However, the improvement of the process did not stop here. As the second attempt was open source, citizens and outside stakeholders have also been able to get involved. The best example of this as follows: one individual made a pull request on Feb 3, 2017 demonstrating how the XGBoost model was finding critical violations, on average, 7.79 days earlier; this represented an improvement on the current model in use. Four days later the Chief Data Officer of Chicago had commented on it and a code review has been initiated and stated, "If the results hold, we will incorporate your contributions to the model that drives food inspections in the city. Thank you and we will be in touch soon" [24]. This provides a clear demonstration of how outside stakeholders are able to play a role in the co-creation of OGD-driven public services. The model is still in use by the CDoPH today and it is still actively maintained by the CDoIT, and stakeholders are still able to suggest improvements to the model through GitHub.

One result of the code being open is that it has been possible for other stakeholders to take, adopt, and change the code for their own uses. The best-known example of this is that of Montgomery Country, Maryland. Montgomery County had hired Open Data Nation, a private sector data analytics company, to take Chicago's code and adopt it for Montgomery County's needs. However, this trial has been stopped due to political reasons. This is an interesting fact, and the reasons why the model is able to work in Chicago, but not other areas, will be discussed in the next section of this paper.

This case is truly interesting as it represents one of the only examples that the authors' found that seems to represent a co-created OGD-driven public service. It is also a service that is able to continue to provide value moving into the future. As more data is generated, the model is likely to become more accurate in predicting critical violations. It will be interesting to follow up on this case in the future to see how the co-creation of the service progressed as well as how the accuracy improves over time. 


\section{Discussion}

This section will discuss what seems to be relevant, and what not, in regards to the current understanding of co-created OGD-driven public services. The discussion aims to reflect back on the conceptual model by comparing it to what emerged in the case. Furthermore, propositions for co-created OGD-driven public services will be put forth.

While conducting the interviews for this case, stakeholders highlighted a multitude of factors that allowed for the co-created OGD-driven public service to be implemented; it was also stated by many interviewees that the process seemed to be a "perfect storm". This "perfect storm" consisted of having external funding, motivated stakeholders, innovative leaders, proper communication channels, an existing OGD portal, and developing the model in an agile way that accepted the fact that mistakes would be made throughout development.

\section{External Funding}

The City of Chicago had received a grant from Bloomberg Philanthropies to develop a "SmartData" portal. It was confirmed by the Chief Data Officer, Tom Schenk, that external funding had allowed the CDoIT to pursue actively more projects, such as the project that this case focuses on. Though external funding does appear to be an active driver for the cocreation of OGD-driven public services, it should be explored further to see what effect it has when a government agency is not the main driving force behind the services.

\section{Motivated Stakeholders}

The model was co-created by numerous different stakeholders representing different sectors; Table 1 presents all stakeholders and their role in the project.

\begin{tabular}{ll}
\hline Stakeholder & Role in Project \\
\hline CDoIT & $\begin{array}{l}\text { Co-Creator of model } \\
\text { Maintains open } \\
\text { government data portal }\end{array}$ \\
\hline CDoPH & Service user \\
\hline $\begin{array}{l}\text { Allstate Insurance } \\
\text { Data Science Group }\end{array}$ & Co-Creator of model \\
\hline CCA & $\begin{array}{l}\text { Initial project manager } \\
\text { Organized Allstate CDoIT } \\
\text { communication }\end{array}$ \\
\hline Citizens & $\begin{array}{l}\text { Model improvements and } \\
\text { pull requests on GitHub }\end{array}$ \\
\hline \multicolumn{1}{c}{$\begin{array}{l}\text { Table 1: Chicago predictive food analytics model } \\
\text { co-creators and their Roles. Source: Authors. }\end{array}$}
\end{tabular}

The model was developed in close cooperation between the CDoIT, Allstate, and $\mathrm{CDoPH}$; this interaction was brokered by the CCA. Interestingly, one interviewee stated that the role of a mediating stakeholder, the CCA, seemed to be quite important. In the interviewee's experience, public and private sector organizations sometimes clash due to organizational differences, but the CCA was able to work as a mediator and help build a bridge and develop the relations between private and public sector. The final group of stakeholders is that of the citizens. As there has been citizen input that improved the efficiency of the current model, it does appear that there is interest and motivation to play a role in the cocreation of OGD-driven public services.

\section{Innovative Leaders}

While conducting interviews, two names were always stated as playing a critical role in the success of the project; Tom Schenk (Chief Data Officer of Chicago) and Gerrin Butler (Director of Food Protection for the City of Chicago). Tom was said to be the main driving force behind the model and had it in mind for the code to be open source since the idea was conceived. Gerrin was the actor who agreed to go ahead with Tom's plan for data-driven food analytics. Gerrin did not initially understand what or how a data analytics model would work and improve current operations, but was willing to try and played an active role throughout. Without the work and willingness of leaders to push for and try new things, this case would not have been possible.

\section{Proper Communication Channels}

There were two iterations of development for the predictive model. The first one failed due to a miscommunication between the $\mathrm{CDoPH}$ and Allstate of how the process of food inspections worked. This was noted down, and in the second iteration, there was a strong emphasis on appropriate communication between parties so that all could be understood. One interesting part of this communication was how technical and non-technical requirements and terminologies were understood and translated by different involved parties. On the $\mathrm{CDoPH}$ side, a list or annex of technical terms was developed so that technical conversations could be followed. On the development side, the requirements were asked for multiple times and a member of the $\mathrm{CDoPH}$ team who had experience in data analytics was able to effectively translate their current process into one more understandable for the data analysts working on the project.

\section{Existing OGD Portal}

Chicago has had an OGD portal since 2010, but it was improved greatly and made a legal requirement in 2012. It was stated by multiple interviewees that the OGD portal allowed them to come up with the idea for the new co-created OGD-driven public service, and that there were no noticeable issues with data quality. It is also important to note that a majority of the open 
data sets that were used in the development of the model were freely accessible to all on the OGD portal. Thus, the OGD portal allowed a new service to be thought up, and it could be created through the exploitation of high quality and easily exploitable OGD sources through Chicago's OGD portal.

\section{Agile Development}

Though the service did not follow traditional agile development methodology, some aspects of agile development were present. The service was developed and tested constantly, improvements were made and tested throughout development, and if mistakes were made, they were learned from and used to improve the service quickly.

\section{The Conceptual Model}

It does appear that there is room for improvement in the model that was proposed in section 2.1 based on the aforementioned factors. One of the first things to address is that it does appear that the model for cocreated OGD-driven public services varies depending on the sector of the stakeholder(s) that are initiating the service. In the case at hand, external funding was one of the major drivers, whereas this may not necessarily be true if a citizen or a company is taking the lead in developing the service, but this should be explored in further research. The role and importance of communication and networks is not currently highlighted in the framework, but from this case, it does appear that communication and understanding between different stakeholders has a large effect on how well the co-creation of an OGD-driven public service goes. However, the case also seems to validate some aspects of the model. When looking at the case it does appear to follow the co-initiation, co-design, co-implementation, and co-evaluation cycle. The model also proposed that OGD might act as a catalyst to drive co-creation of OGD-driven public services; this also seems to be supported by this case. The Allstate team specifically chose the subject for this case as there was OGD available, and this data was easily accessible, exploitable, and of high quality.

\section{Propositions}

1. In an environment where open government data and tools for data analytics, exploitation and co-creation are made widely available, any actor can take the lead and initiate or co-create data-driven services that create public value.

2. When OGD is released and maintained, it allows the Government to act as a platform. This platform allows OGD sets to be exploited and leads to increased levels and occurrences of co-created OGD-driven public services.

3. A "perfect storm" consisting of sufficient resources, innovative leaders, motivated stakeholders, and access to OGD allows for effective execution of co-created OGD-driven public services.

4. Co-created OGD-driven public services appear to have the potential to drive increased levels of efficiency traditionally slow or outdated processes.

5. Government as a Platform appears to be a bridge that allows for the concepts of cocreation and OGD to be merged together. If the government makes data available, and this data is used to create a new public service, then at a minimal level there will always be co-creation between the government and the one exploiting the data for the OGD-driven public services.

From the case, six different factors were highlighted that seem to play an important role in the co-creation of OGD-driven public services. After presenting these factors they were used to reflect back on our current understanding of co-created OGD-driven public services and the model provided in section 2.1. After this reflection 5 propositions have been proposed that deal with how co-creation of OGD-driven public services occurs, how the idea of GaaP leads to cocreating of OGD-driven public services, as well as what benefits a co-created OGD-driven public service may have. 


\section{Conclusion}

The aim of this paper was to present the case of Chicago's predictive food analytics model so that new insights into the concept of co-created OGD-driven public services could potentially emerge. In section 3.1 , the context surrounding the case was presented, specifically, the role that external funding, a functioning OGD portal, and previous experience with OGD-based predictive analytics. These three factors seem to have played an instrumental role in laying the foundation for the co-creation of OGD-driven public services in the city of Chicago. Chicago appears to have a 'platform' that is based on their OGD portal, this government platform thus allows for the exploitation and co-creation of new OGD-driven public services.

On the private sector side, an interesting policy was discovered. Allstate Insurance's "bluelight" policy allowed their employees to participate in pro bono data science work, thus providing the opportunity for their staff to engage in co-creation with the City of Chicago. The role of Allstate also seems to demonstrate that there is interest from those with experience in data science to participate in pro bono work and in the co-creation of new OGD-driven public services.

Section 4 provided a discussion on the findings from the case. Firstly, six factors were outlined as playing a key role in allowing the co-creation of an OGD-driven public service to take place: external funding, motivated stakeholders, innovative leaders, proper communication channels, an existing OGD portal, and agile development practices. These factors were then used to reflect back on one proposed conceptual model for how the process of co-created OGD-driven public services is understood. These reflections allow for potential improvements to the conceptual model to be made, but it also allows some preliminary validation to take place of the model. It does appear that the idea of co-created OGD-driven public services has merit and does exist in the real world. The way in which the service was developed in Chicago also seems to match the four stages that were proposed in the conceptual model. The final part of the discussion was the presentation of some initial propositions on co-created OGD-driven public services. These propositions may be briefly summarized as follows, availability of OGD and tools for data analytics has the potential to enable the cocreation of OGD-driven public services, governments releasing OGD are acting as a platform and from this platform the co-creation of new and innovative OGDdriven public services may take place, and that the idea of GaaP does appear to be an idea that allows for the topics of co-creation and OGD to be merged together.

Though the case presented in this paper represents an empirical example of a co-created OGD-driven public service, it only represents one possible combination of stakeholder roles as a governmental agency was still playing a major role. As the notion of a co-created OGD-driven public service implies that the government need not play an active role in the development, any examples of co-created OGDdriven public services where a non-traditional stakeholder is playing a leading role could provide valuable insight into the formulation of the understanding of co-created OGD-driven public services. Secondly, this case study also only looks at one type of a co-created OGD-driven public service (a data analytics model), other types of services may exist (such as web or mobile applications built on OGD), and research should be further conducted on the different types of co-created OGD-driven public services.

The exploratory case study that was conducted for this paper provides an initial empirical case on a cocreated OGD-driven public service and aims to advance and encourage research into the topic of cocreation of OGD-driven public services. The case demonstrates that there is a link between co-creation and OGD, and that this link may enable or drive a change in the current understanding of public services. Furthermore, the case also demonstrates that there is a relationship between GaaP and OGD and that this relationship is likely to encourage or enable cocreation. This paper provides an initial stepping-stone on the topic of co-created OGD-driven public services and, as such, proposes that future research into the topic is needed. Potential avenues of future research include solidifying the definition of a co-created OGD-driven public service, empirical work focusing on different types of co-created OGD-driven public services, studies that aim to understand the role that different stakeholders as the leading service developer have on the co-creation process of OGD-driven public services, and also how the idea of GaaP influences our understanding of co-created OGD-driven public services and the bridge between OGD and co-creation.

Acknowledgements. This work was supported by the European Commission (OpenGovIntelligence H2020 grant 693849) and Estonian Research Council (PUT773, PUT1361). 


\section{References}

[1] G. Galasso, G. Garbasso, G. Farina, T. Kalvet, F. Mureddu, D. Osimo, and P. Waller, Analysis of the value of new generation of eGovernment services (SMART 2014/066). 2016.

[2] M. Janssen, Y. Charalabidis, and A. Zuiderwijk, "Benefits, Adoption Barriers and Myths of Open Data and Open Government," Inf. Syst. Manag., vol. 29, no. 4, pp. 258-268, 2012.

[3] A. Zuiderwijk, M. Janssen, S. Choenni, R. Meijer, and R. S. Alibaks, "Socio- technical Impediments of Open Data," Electron. J. Electron. Gov., vol. 10, no. 2, pp. 156-172, 2012.

[4] M. Toots, K. McBride, T. Kalvet, and R. Krimmer, "Open Data as Enabler of Public Service Co-creation : Exploring the Drivers and Barriers," in Proceedings of the 2017

International Conference for E-Democracy and Open Government (CeDEM 2017). IEEE Computer Society, pp. 102-112, 2017.

[5] E. Ostrom, "Metropolitan reform: Propositions derived from two traditions," Soc. Sci. Q., pp. 474-493, 1972.

[6] M. Toots, K. McBride, T. Kalvet, R. Krimmer, E. Tambouris, E. Panopoulou, E. Kalampokis, and K. Tarabanis, "A Framework for Data-Driven Public Service Co-Production," in Electronic Government: Proceedings of the 16th IFIP WG 8.5 International Conference, EGOV 2017, M. Janssen et al., Eds. Springer, pp. 264-275, 2017.

[7] European Commission, "A vision for public services,” p. 16, 2013.

[8] K. McBride, "Government as a Platform: Exploiting Open Government Data to Drive Public Service Co-Creation," Tallinn University of Technology, 2017.

[9] D. Linders, "From e-government to wegovernment: Defining a typology for citizen coproduction in the age of social media," Gov. Inf. $Q$. , vol. 29, no. 4, pp. 446-454, 2012.

[10] V. Lember, "The role of new technologies in coproduction," in Co-production and co-creation: engaging citizens in public service delivery., $\mathrm{T}$. Brandsen, T. Steen, and B. Verschuere, Eds. Routledge, forthcoming in 2018.

[11] M. Kassen, "A promising phenomenon of open data: A case study of the Chicago open data project," Gov. Inf. Q., vol. 30, no. 4, pp. 508-513, 2013.

[12] T. Schenk, "Food Inspection Forecasting - City of Chicago." [Online]. Available: https://chicago.github.io/food-inspectionsevaluation/. [Accessed: 21-Apr-2017].

[13] R. K. Yin, Case study research: Design and methods, Rev. Newbury Park, Calif.: Sage publications, 2013.

[14] Open Data Executive Order (No. 2012-2), City of Chicago, 2012.
[15] "City of Chicago Developers," City of Chicago Open Data Developer Portal. [Online]. Available: http://dev.cityofchicago.org/. [Accessed: 13-Jun2017].

[16] B. Goldstein, "Open Data in Chicago: Game On," Beyond Transparency, 2013. [Online]. Available: http://beyondtransparency.org/chapters/part1/open-data-in-chicago-game-on/. [Accessed: 13Jun-2017].

[17] "Bloomberg Philanthropies Announces Mayors Challenge Winners Providence, Chicago, Houston, Philadelphia, and Santa Monica," Bloomberg Philanthropies, 2013. [Online]. Available: https://www.bloomberg.org/press/releases/bloomb erg-philanthropies-announces-mayors-challengewinners-providence-chicago-houstonphiladelphia-and-santa-monica/. [Accessed: 13Jun-2017].

[18] Ash Center Mayors Challenge Research Team, "Chicago's SmartData Platform - Pioneering Open Source Municipal Analytics," Data-Smart City Solutions, 2014. [Online]. Available: http://datasmart.ash.harvard.edu/news/article/chica go-mayors-challenge-367. [Accessed: 13-Jun2017].

[19] S. Thornton, "Using Predictive Analytics to Combat Rodents in Chicago | Data-Smart City Solutions," Data-Smart City Solutions, 2013. [Online]. Available: http://datasmart.ash.harvard.edu/news/article/using -predictive-analytics-to-combat-rodents-inchicago-271. [Accessed: 13-Jun-2017].

[20] "Restaurant Inspection," City of Chicago, 2017. [Online]. Available: https://www.cityofchicago.org/city/en/depts/cdph/ provdrs/inspections_and_permitting/svcs/food_pro tection_program.html. [Accessed: 13-Jun-2017].

[21] "Civic Consulting Alliance," CCA, 2017. [Online]. Available: http://www.ccachicago.org/. [Accessed: 13-Jun-2017].

[22] S. Thornton, "Delivering Faster Results with Food Inspection Forecasting - Chicago's AnalyticsDriven Plan to Prevent Foodborne Illness," DataSmart City Solutions, 2015. [Online]. Available: http://datasmart.ash.harvard.edu/news/article/deliv ering-faster-results-with-food-inspectionforecasting-631. [Accessed: 13-Jun-2017]. 